\title{
Exploiting Polarization for Spectrum Sensing in Cognitive SatComs
}

\author{
Shree Krishna Sharma, Symeon Chatzinotas, and Björn Ottersten \\ SnT - securityandtrust.lu, University of Luxembourg \\ Email:\{shree.sharma, symeon.chatzinotas, bjorn.ottersten\}@uni.lu
}

\begin{abstract}
Exploring new techniques for spectrum sensing (SS), which can detect the weak primary signals instantly, has been an important research challenge. In this paper, the problem of enhancing SS efficiency in cognitive SatComs has been considered. The analysis of different combining techniques has been carried out for SS using dual polarized antenna. Furthermore, polarization states of received signals are exploited and based on obtained polarization states, Optimum Polarization Based Combining (OPBC) technique has been used for carrying out $\mathrm{SS}$ in the satellite terminal. The sensing performance of OPBC technique has been compared to selection combining (SC), equal gain combining (EGC) and maximum ratio combining (MRC) techniques. The simulation results show that OPBC technique achieves a great improvement in sensing efficiency over other considered techniques at the expense of complexity in a dual polarized AWGN channel.
\end{abstract}

Index Terms-probability of detection, sensing efficiency, energy detection, dual polarized antenna, polarization states

\section{INTRODUCTION}

Spectrum sensing is one important mechanism for the spectrum awareness required by cognitive radios (CR). In terms of $\mathrm{CR}, \mathrm{SS}$ involves obtaining the spectrum usage characteristics across multiple dimensions such as time, space, frequency, and code. It also determines which type of signal is occupying the spectrum including different characteristics such as modulation type, waveform, bandwidth, carrier frequency etc. Existing SS literature mainly considers the use of amplitude, frequency or phase characteristics of the signals, which can be referred as scalar signal processing [1]. Among many available SS techniques in the literature, energy detection (ED) is the most common way of SS because of its low computational and implementation complexities [2,3]. The electromagnetic (EM) signal propagating through space is polarized with some polarization state. The polarization state of a signal, a basic vector characteristic of an EM signal, is the orientation of the electric field vector at a given point in space, during one period of oscillation. Since traditional ED techniques only consider amplitude information of one component of signal and reject the information from another orthogonal component, information provided by the vector nature of EM signals is not fully exploited.

The concepts of spectrum sharing in the frequency, time and spatial domains have been considered in much literature but the polarization domain has not been fully exploited. In existing literature, the polarization domain has been used for diversity and multiplexing purposes [4-6]. The use of the polarization domain for SS has not been investigated extensively and few research works have been reported in literature. The concept of virtual polarization adaption for increasing the detection capability of a radar system by using vector signal processing has been proposed in [1]. A Virtual Polarization Detection (VPD) method has been proposed in [7] as a means to enhance SS performance of Secondary Users (SUs), making use of vector polarization states. A polarization sensing technique exploiting the transmit and receive polarization states has been proposed in [8], which takes into account the interference constraint of PU and the signal to interference noise ratio (SINR) demands of SUs. An optimal polarization receiving (OPR) scheme for CRs has been proposed in [9] to improve the SINR demands of SUs.

Various cognitive techniques have been investigated in the literature. The most common cognitive techniques can be categorized into interweave or SS, underlay, and overlay techniques [10]. In SS only techniques, SUs are allowed to transmit whenever primary users (PUs) do not use that particular band, whereas in underlay techniques, SUs are allowed to transmit as long as they meet the interference constraint of PUs. SS with an underlay technique considered in this work detects the presence or absence of PU and SUs are permitted to transmit in other polarization states than that of PUs even if a PU is present. Although it is possible to compare these two different cognitive techniques in terms of spectral efficiency, this comparison is not the focus of this paper.

In this work, the polarization domain has been exploited as an additional degree of freedom to explore efficient SS and transmission schemes in the context of cognitive satellite communications (SatComs) using the concept of VPD technique introduced in [1]. Generally, Fixed Service Satellites (FSS) and terrestrial base station (BS) antennas use horizontal $(\mathrm{H})$ and vertical (V) polarization, whereas Direct Broadcast Satellites (DBS) may use left and right hand circular polarization as well as $\mathrm{H} / \mathrm{V}$ polarization. The state of polarization is important in determining the energy transfer efficiency between transmit and receive antenna [11]. By deploying two orthogonally polarized antennas, any type of receive or transmit polarization can be derived. Detecting the polarization state in addition to energy of a certain carrier frequency can significantly increase the spectrum efficiency by investigating suitable cognitive techniques in polarization domain. Different combining techniques considered in this paper exploit both H-polarized and V-polarized components using vector signal processing. Since 
a dual polarized antenna receives both polarized components but it receives noise with identical average power, increased SS performance can be expected because of better signal reception.

The rest of this paper has been organized as follows: section II presents the use cases, where the technique considered in this paper can be applied. Section III provides the theoretical basis of traditional ED technique in the context of single antenna. Section IV describes the ED problem in the context of dual polarized antennas. SC, EGC, and MRC techniques for SS are discussed in section V. Section VI describes the OPBC technique using polarization state information of the received signal. Section VII describes the simulation environment and simulation results. Section VIII concludes this paper by summarizing the results.

\section{USE CASES}

One cognitive scenario can be a terrestrial microwave system as a primary link and satellite uplink transmission from a satellite terminal as a secondary link. In this scenario, the satellite terminal can be considered to be deployed with dual polarized antenna and the primary terrestrial link as a strong line of sight (LOS) link. This scenario can be beneficial for satellite operators to take advantage of terrestrial licensed spectrum in secondary basis. To reduce complexity, same dual polarized antenna can be used for sensing and transmission purposes. Two radiators of dual polarized antenna at the satellite terminal detect H-polarized and V-polarized signals, which can be used to find out the polarization states of primary signals coming to the satellite terminal. On the basis of detected polarization states, the satellite terminal can transmit in the polarization state other than that of detected polarization states. In this way, satellite uplink transmission can use the same spectrum as used in microwave LOS link in secondary basis. This scenario has been shown in Fig. 1 . The interference from terrestrial BS antenna to the satellite can be considered to be negligible, whereas interference from satellite terminal to terrestrial BS antenna is strong and needs to be taken into account to meet the interference constraint of primary terrestrial users.

Another application scenario can be a dual satellite cognitive scenario, as shown in Fig. 2, consisting of two satellites equipped with multi-beam antennas and providing coverage to same geographic area. It can be assumed that frequency reuse concept is used to increase the capacity and cognitive satellite terminal may be considered to be equipped with circularly polarized antenna. The cognitive satellite terminal to be connected to the secondary satellite within a particular beam can sense the polarization used by primary satellite and uses the opposite sense of polarization to transmit its data using the same carrier frequency in secondary basis.

\section{ENERGY DETECTION FOR SINGLE ANTENNA CASE}

The ED problem was first studied in [12] for deterministic signals transmitted over a flat band-limited Gaussian noise channel. The ED problem for signals transmitted over a variety

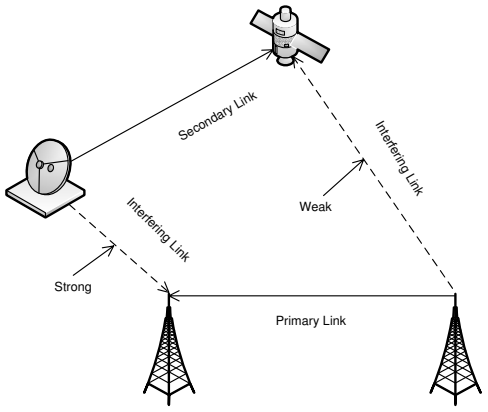

Fig. 1: Hybrid satellite terrestrial cognitive scenario

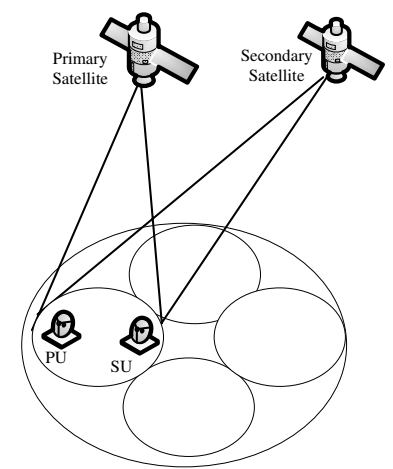

Fig. 2: Dual satellite cognitive scenario

of fading channels has been considered in [2] and [13]. The problem analysis is further extended to include various receive diversity schemes in [14]. In [15], comprehensive performance analysis of the energy detector over fading channels with single antenna reception and with antenna diversity has been developed. The problem of PU detection using ED technique in AWGN channel can be presented in the form of following binary hypothesis testing problem.

$$
\begin{aligned}
& H_{0}: x(t)=n(t) \quad \mathrm{PU} \text { absent } \\
& H_{1}: x(t)=s(t)+n(t) \quad \mathrm{PU} \text { present }
\end{aligned}
$$

where $n(t)$ is bandpass noise signal, can be represented as:

$$
n(t)=n_{i}(t) \cos 2 \pi f_{c} t-n_{q}(t) \sin 2 \pi f_{c} t
$$

where $f_{c}$ is the reference frequency, $n_{i}(t)$ is the in-phase component and $n_{q}(t)$ is the quadrature phase component. If bandpass noise is considered within bandwidth $B, n_{i}(t)$ and $n_{q}(t)$ have bandwidth $B / 2$ and variances of $n_{i}(t), n_{q}(t)$ and $n(t)$ are all equal to the noise power. The noise energy in a sample of finite duration $T$ can be approximated as [12]:

$\bar{Y}=\int_{0}^{T} n^{2}(t) d t \approx \frac{1}{2 B} \sum_{m=1}^{T B}\left(a_{i m}{ }^{2}+a_{q m}{ }^{2}\right)=N_{o} \sum_{i=1}^{T B}\left(b_{i m}{ }^{2}+b_{q m}{ }^{2}\right)$

where $a_{i m}$ and $a_{q m}$ are $m$-th samples taken at the interval of $\frac{1}{B}$ of $n_{i}(t)$ and $n_{q}(t)$ respectively, $b_{i m}=a_{i m} / \sigma_{i}^{2}$ and $b_{q m}=$ $a_{q m} / \sigma_{i}^{2}, \sigma_{i}^{2}=\operatorname{var}\left(a_{i m}\right)=\operatorname{var}\left(a_{q m}\right)=2 N_{o} B$ and $N_{o}$ is two sided power spectral density (PSD) of noise. Denoting $\bar{Y} / N_{o}$ 
by $Y$, the following equation can be obtained:

$$
Y \approx \sum_{m=1}^{T B} b_{i m}{ }^{2}+\sum_{m=1}^{T B} b_{q m}{ }^{2}
$$

From the above equation, it can be inferred that the decision statistic $Y$ follows a central chi-square $\chi^{2}$ distribution with $2 T B$ degrees of freedom under hypothesis $H_{0}$. Under hypothesis $H_{1}$, signal and noise both are present and decision statistic $Y$ follows a non-central chi-square $\chi^{2}$ distribution with $2 T B$ degrees of freedom (dof) and non-centrality parameter $\bar{\gamma}[16]$, i.e.

$$
Y \sim \begin{cases}\chi_{2 T B}^{2}, & H_{0} \\ \chi_{2 T B}^{2}(\bar{\gamma}), & H_{1}\end{cases}
$$

The probability of false alarm $\left(P_{f}\right)$ and probability of detection $\left(P_{d}\right)$ can be computed by [2];

$$
\begin{aligned}
& P_{f}=\operatorname{Pr}\left(Y^{\prime}>\lambda_{t h} \mid H_{0}\right), \\
& P_{d}=\operatorname{Pr}\left(Y^{\prime}>\lambda_{t h} \mid H_{1}\right)
\end{aligned}
$$

where $\lambda_{t h}$ is the decision threshold. The expression for $P_{f}$ can be written as :

$$
P_{f}=\frac{\Gamma\left(u, \frac{\lambda_{t h}}{2}\right)}{\Gamma(u)}
$$

where $\Gamma(.,$.$) is the incomplete gamma function, \Gamma($.$) is the$ complete gamma function, and $u=T B$. The expression for $P_{d}$ can be written as:

$$
P_{d}=Q_{u}\left(\sqrt{\bar{\gamma}}, \sqrt{\lambda_{t h}}\right)
$$

where $\bar{\gamma}=2 T B \gamma, \gamma$ being signal to noise power ratio (SNR) of the PU signal and $Q_{u}(.,$.$) is the generalized Marcum Q-$ function.

\section{ENERGY DETECTION FOR DUAL POLARIZED ANTENNA}

Two branches of dual polarized antenna can be considered to receive $\mathrm{H}$-polarized and $\mathrm{V}$-polarized signals coming from $\mathrm{PU}$ transmit antenna to the SU receive antenna. In the considered use cases, a single SU and a single PU can be considered for the sake of simplicity. Let $\mathbf{s}_{p}$ be PU transmit vector signal and $\mathbf{y}_{p s}$ be the signal vector arriving at the dual polarized antenna of SU. The transmit signal vector $\mathbf{s}_{p}$ can be generated from H-polarized component $s_{p H}$ and V-polarized component $s_{p V}$, which are orthogonal to each-other. Let us consider that $\mathbf{X}_{t}$ and $\mathbf{X}_{r}$ are polarization states of primary transmit and receive antennas respectively, and $\mathbf{C}_{t}$ and $\mathbf{C}_{r}$ are polarization states of secondary transmit and receive antennas respectively. The transmit signal vector $\mathbf{s}_{p}$ can be represented in terms of Jones vector as [7]:

$$
\mathbf{s}_{p}=\left[\begin{array}{c}
s_{p H} \\
s_{p V}
\end{array}\right]=\left[\begin{array}{c}
s_{p H}^{i}+s_{p V}^{q} \\
s_{p H}^{i}+s_{p V}^{q}
\end{array}\right]
$$

where $i$ and $q$ are in-phase and quadrature phase components. Then the polarization state of transmit antenna in terms of Jones vector can be written as [8]:

$$
\mathbf{X}_{t}=\left[\begin{array}{c}
\cos \alpha_{p} \\
\sin \alpha_{p} e^{j \phi_{p}}
\end{array}\right]
$$

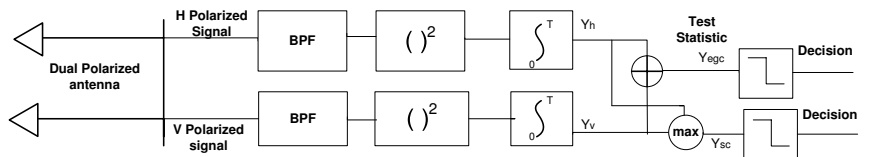

Fig. 3: Block diagram of SC and EGC techniques for SS

where $\alpha_{p} \in(0, \pi / 2), \phi_{p} \in(0,2 \pi)$ can be calculated using the following equations.

$$
\begin{aligned}
\alpha_{P} & =\arctan \frac{\left|s_{p V}\right|}{\left|s_{p H}\right|} \\
\phi_{p} & =\phi_{p V}-\phi_{p H}=\arctan \frac{\left|s_{p V}^{q}\right|}{\left|s_{p V}^{i}\right|}-\arctan \frac{\left|s_{p H}^{q}\right|}{\left|s_{p H}^{i}\right|}(11)
\end{aligned}
$$

The components $s_{p H}$ and $s_{p V}$ can then be weighted by using a power imbalance factor $a=\tan \left(\alpha_{p}\right)$ to impact the practical scenario of power imbalance in two branches of dual polarized antenna in the following way to make total energy unity.

$$
\mathbf{s}_{p}=\left[\begin{array}{c}
\frac{1}{1+a^{2}} s_{p H} \\
\frac{a^{2}}{1+a^{2}} s_{p V}
\end{array}\right]
$$

Assuming that the channel conditions between PU and SU remain ideal during the observation time interval $t$, the detection problem can be stated in terms of following binary hypothesis.

$$
\begin{aligned}
& H_{0}: \mathbf{y}_{p s}(t)=\mathbf{n}(t) \quad \text { PU absent } \\
& H_{1}: \mathbf{y}_{p s}(t)=\mathbf{s}_{p}(t)+\mathbf{n}(t) \quad \text { PU present }
\end{aligned}
$$

where $\mathbf{n}(t)$ represents the zero mean complex Gaussian noise vector. The decision statistic to check above hypothesis problem depends on the type of combining technique used in combining H-polarized and V-polarized signals.

\section{COMbining TeCHNiQues FOR DUAL POLARIZED ANTENNA}

\section{A. Selection Combining}

The block diagram of SC technique for SS using dual polarized antenna has been shown in Fig. 3. The H-polarized and V-polarized received signals are passed through the bandpass filter (BPF) to limit the noise power and then passed through squared and integrator devices. In this combining technique, combined signal to noise ratio (SNR) is the maximum of the branch SNRs. With SC technique, the receiver selects the antenna with the highest received signal power and eliminates observations from another antenna [17]. The decision statistic for SC is based on the following condition.

$$
Y_{s c}=\max \left(Y_{h}, Y_{v}\right)
$$

since $Y_{h}$ and $Y_{v}$ are decision statistics for H-polarized branch and V-polarized branch respectively. The expression for $P_{f}$ becomes same as (7) and the expression for $P_{d}$ can be written as:

$$
P_{d}=Q_{u}\left(\sqrt{\bar{\gamma}_{s c}}, \sqrt{\lambda_{t h}}\right)
$$

where $\bar{\gamma}_{s c}=2 T B \gamma_{s c}, \gamma_{s c}$ being SNR of the PU signal after performing SC. 


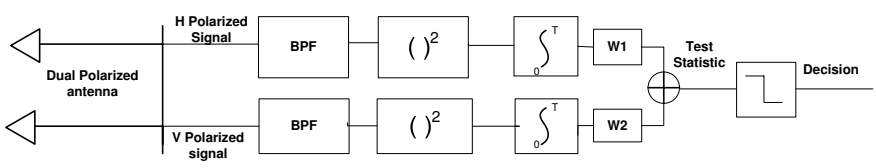

Fig. 4: Block diagram of MRC technique for SS

\section{B. Equal Gain Combining (EGC)}

The block diagram of EGC technique has been shown in Fig. 3. This technique sets unit gain in each branch and removes the problem of requirement of varying weights as required in MRC technique. This method is a trade-off between SC and MRC methods [17]. This is the case of equal gain combining of two branches with unequal SNR values since each polarized signal carries different amount of transmitted signal power. The final decision statistic using EGC can be written as:

$$
Y_{e g c}=Y_{h}+Y_{v}
$$

The decision statistic $Y_{e g c}$ under hypothesis $H_{1}$ follows a central chi-square $\chi^{2}$ distribution with $2 T B$ degrees of freedom with non-centrality parameter $\bar{\gamma}_{e g c}$, which can be written as:

$$
\bar{\gamma}_{e g c}=\bar{\gamma}_{h}+\bar{\gamma}_{v}
$$

where $\bar{\gamma}_{h}$ and $\bar{\gamma}_{v}$ are non-centrality parameters corresponding to received signals from $\mathrm{H}$-polarized and $\mathrm{V}$-polarized branches respectively. The decision statistic $Y_{e g c}$ is then compared with a preset threshold $\lambda_{t h}$ and hypothesis $H_{1}$ is declared if $Y_{e g c}>\lambda_{t h}$ and hypothesis $H_{0}$ is declared if $Y_{e g c}<\lambda_{t h}$. The expression for $P_{f}$ become same as equation (7) and expression for $P_{d}$ can be written as:

$$
P_{d}=Q_{u}\left(\sqrt{\bar{\gamma}_{e g c}}, \sqrt{\lambda_{t h}}\right)
$$

where $\bar{\gamma}_{e g c}=2 T B \gamma_{e g c}, \gamma_{e g c}$ being SNR of PU signal after performing EGC.

\section{Maximum Ratio Combining}

This technique maximizes the output SNR by combining the branch SNRs with different weights [17]. The branch with higher SNR is weighted with greater factor than the branch with lower SNR value. The decision statistic using MRC technique can be written as:

$$
Y_{m r c}=w_{1} Y_{h}+w_{2} Y_{v}
$$

where $w_{1}$ and $w_{2}$ are the weights applied to decision metrics $Y_{h}$ and $Y_{v}$ respectively. The decision statistic $Y_{m r c}$ under hypothesis $H_{1}$ follows a central chi-square $\chi^{2}$ distribution with $2 T B$ degrees of freedom with non-centrality parameter $\bar{\gamma}_{m r c}$, which can be written as:

$$
\bar{\gamma}_{m r c}=2 \frac{w_{1}}{\left(w_{1}+w_{2}\right)} \bar{\gamma}_{h}+2 \frac{w_{2}}{\left(w_{1}+w_{2}\right)} \bar{\gamma}_{v}
$$

The expression for $P_{f}$ become same as (7) and the expression for $P_{d}$ can be written as:

$$
P_{d}=Q_{u}\left(\sqrt{\bar{\gamma}_{m r c}}, \sqrt{\lambda_{t h}}\right)
$$

where $\bar{\gamma}_{m r c}=2 T B \gamma_{m r c}, \gamma_{m r c}$ being SNR of the PU signal after performing MRC.

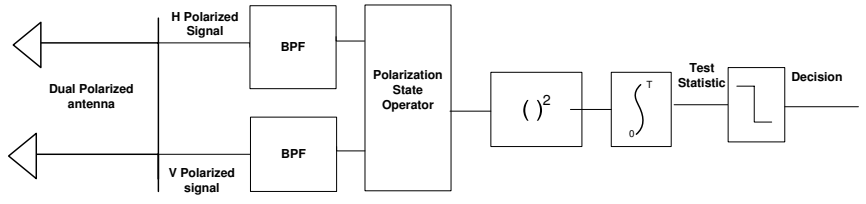

Fig. 5: Block diagram of OPBC technique for SS

\section{OPtimum Polarization BASEd COMBining}

In scalar signal sensing methods such as energy detection, vector signals of PUs are converted to scalar quantities, whereas, in OPBC technique, both $\mathrm{H}$ and $\mathrm{V}$ components of primary signals are combined based on the polarization states of received signals. The signal power at receiving antenna becomes maximum for an incoming field if the receiver has conjugate polarization state as the transmitter and it becomes minimum at orthogonal polarization state to that at transmitter [9]. The block diagram of OPBC technique has been shown in Fig. 5. In cognitive SatCom scenario, polarization state can be adjusted as follows: transmit polarization state of a satellite terminal can be chosen in order to protect the terrestrial PUs from interference caused by it and then its receive polarization state can be optimized to ensure its QoS, mitigating interference from terrestrial PUs to its receiver.

In practical cognitive scenarios, there may be presence of multiple PUs and SU needs to adapt its receiving polarization state $\mathbf{C}_{r}$ to receive different PU signals with different polarization states. Since it is not practical to change the hardware structure of antenna to adapt to different polarization states, virtual polarization adaptation scheme can be used to get the same phenomena as stated in [1]. In this scheme, the polarization adaptation takes place within the processor not in the antenna hardware. It can be noted that OPBC technique involves per-sample processing in contrast to other combining techniques and it needs one combining operation per sensing period. The horizontal and vertical reception of vector signal $\mathbf{y}_{p s}$ through dual polarized antenna can be written in terms of Jones vector as:

$$
\mathbf{y}_{p s}=\left[\begin{array}{l}
y_{p s H} \\
y_{p s V}
\end{array}\right]
$$

The received signal vector $\mathbf{y}_{p s}$ can then be weighted by polarization state vector, which is constrained by power. Polarization state vector can be constructed by multiplying phase weighting operator $\mathbf{P}$ and amplitude weighting operator A, which are given by [7];

$$
\begin{aligned}
& \mathbf{P}=\left[\begin{array}{cc}
1 & 0 \\
0 & e^{j \phi_{s}}
\end{array}\right] \\
& \mathbf{A}=\left[\begin{array}{l}
\cos \alpha_{s} \\
\sin \alpha_{s}
\end{array}\right]
\end{aligned}
$$

The polarization state of $\mathrm{SU}$ receive antenna $\mathbf{C}_{r}$ can be considered to be equivalent to the following form:

$$
\mathbf{C}_{r}=\mathbf{P A}=\left[\begin{array}{c}
\cos \alpha_{s} \\
\sin \alpha_{s} e^{j_{s}}
\end{array}\right]
$$


where $\alpha_{s}$ and $\phi_{s}$ are parameters defining the polarization state and can be calculated from (11) by replacing $\mathbf{s}_{p}$ with $\mathbf{y}_{p s}$. The decision for the presence or absence of PU signal can be made based on the following decision metric.

$$
Y=\sum_{i=1}^{M}\left|\mathbf{C}_{r}^{\dagger} \mathbf{y}_{p s}\right|^{2} \gtrless_{H_{0}}^{H_{1}} \lambda_{t h}
$$

where $M$ is the number of samples and $\dagger$ is the complex conjugate transpose. The value of threshold $\lambda_{t h}$ can be determined by using the preset value of probability of false alarm $\left(P_{f}\right)$. Since $\mathbf{y}_{p s}$ is a complex Gaussian vector and $\mathbf{C}_{r}$ is a linear transformation, the decision statistic $Y$ follows $\chi^{2}$ distribution [16]. The expression for $P_{f}$ become same as (7) and the expression for $P_{d}$ can be written as:

$$
P_{d}=Q_{u}\left(\sqrt{\bar{\gamma}_{o p b c}}, \sqrt{y_{t h}}\right)
$$

where $\bar{\gamma}_{o p b c}=2 T B \gamma_{o p b c}, \gamma_{o p b c}$ being received SNR obtained after applying polarization state operator.

\section{RESUlTS AND DISCUSSION}

\section{A. Simulation Results}

We consider the following six different cases for comparing the sensing performance. 1) First case considers the omnidirectional antenna, which senses the signals coming from all directions independent of polarization state of received signal. 2) Second case considers dual polarized antenna. One radiator of dual polarized antenna is dedicated to receive only $\mathrm{H}$-polarized signal and another dedicated to receive only Vpolarized signal. The received signals from these two different antennas are considered for detection individually. 3) Third case considers SC technique to combine the signals received from two radiators of dual polarized antenna. 4) Fourth case considers EGC technique. 5) Fifth case considers MRC technique. 6) Sixth case considers OPBC technique using the polarization states of received signals.

In the considered simulation environment, two orthogonal polarized signals are generated and they are weighted with variable $a=\tan \left(\alpha_{p}\right)$. Since $\alpha_{p} \in(0, \pi / 2)$, the value of $a$ ranges from 0 to $\infty$. The value of variable $a$ determines the power levels of two orthogonally polarized signals and it is responsible for determining whether the resultant signal is horizontal or vertical or near to any of these polarization states. For example: when $a=1$, both $\mathrm{H}$ and $\mathrm{V}$ components have same half power and when $a=0$, the signal transmitted from transmit antenna is totally H-polarized, Vpolarized signal being absent. Dual polarized AWGN channel has been considered due to lack of scattering in SatComs. Fig. 6 shows the comparison of Receiver Operating Characteristic (ROC) curves for six different cases at SNR value of $-2 \mathrm{~dB}$, degree of freedom (dof) value 4 and $10^{4}$ number of iterations (N). The value of $\alpha_{p}$ in this case is $\pi / 6$ and corresponding value of power imbalance factor $a$ is 0.5777 . The dof value determines the time bandwidth product i. e. it is equal to $2 T B$. The received SNR of single omnidirectional antenna has been considered to be half than that of dual polarized antenna to

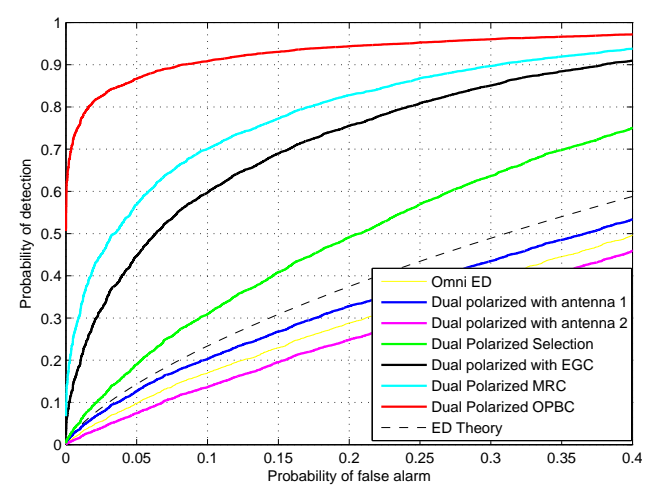

Fig. 6: Comparison of ROC curves for different combining techniques

carry out fair comparison between single antenna and two antenna cases. From the simulation results, it can be observed that OPBC technique performs much better than all the other cases and MRC performs slightly better than EGC and EGC performs better than SC technique. SC performs better than case 1 and case 2 due to selection of larger decision metric in each iteration while calculating $P_{d}$. Moreover, the sensing performance of dual polarized with antenna 1 is observed to be better than that of single omnidirectional antenna case and dual polarized with antenna 2 because antenna 1 in this case is receiving higher strength signal than antenna 2 due to power imbalance factor $a$ applied to two orthogonal signals. Theory ED curve has been drawn using (7) and (8) as a reference. Furthermore, it has been noted during simulation that theory ED curve closely matches with single antenna case when full received SNR has been considered.

Fig. 7 shows comparison of different cases in terms of Complementary Receiver Operating Characteristic (CROC) curves $\left(\mathrm{SNR}=5 \mathrm{~dB}, \mathrm{dof}=6, \alpha_{p}=\pi / 6, \mathrm{~N}=3 \times 10^{5}\right)$. It can be observed from figure that for the same $P_{f}$, probability of miss $\left(P_{m}=1-P_{d}\right)$ is much less in case 6 than in other 5 cases and it increases while going from case 6 to case 1 for the same value of $P_{f}$.

Fig. 8 shows comparison of CROC curves of considered 3, 4,5 and 6 cases in terms of different values of SNR ( $\mathrm{dof}=6$, $\alpha_{p}=\pi / 6, \mathrm{~N}=3 \times 10^{5}$ ). SNR values of $-2 \mathrm{~dB}$ and $5 \mathrm{~dB}$ have been considered for comparison. It can be observed from the figure that the probability of missing the PU presence for each case gets decreased as SNR is increased. Fig. 9 shows the plot of $P_{d}$ versus $\alpha_{p}$ angle $\left(\mathrm{SNR}=-2 \mathrm{~dB}, \mathrm{dof}=4, \mathrm{~N}=10^{4}\right)$. The range of $\alpha_{p}$ has been taken from 0 to $\pi / 2$ and the value of $P_{f}$ has been taken as 0.1. It can be observed from the result that $P_{d}$ with dual polarized antenna 1 exceeds the value of $P_{d}$ of dual polarized antenna 2 up to the value of $\pi / 4$ and the relation gets reverse after this value. Furthermore, it can be observed that $P_{d}$ versus $\alpha_{p}$ curve becomes approximately flat for SC, EGC, MRC and OPBC cases i.e. $P_{d}$ is independent of value of $\alpha_{p}$.

\section{B. Discussion}

From the simulation results, it can be observed that for the same value of $P_{f}$ and SNR, OPBC technique can sense the 


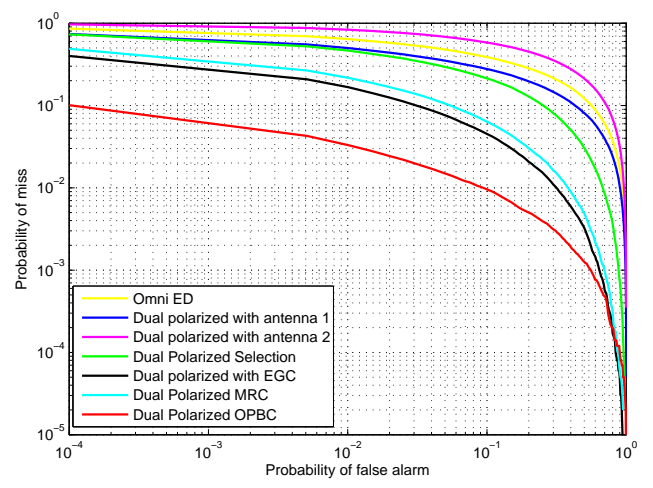

Fig. 7: Comparison of CROC curves for different combining techniques

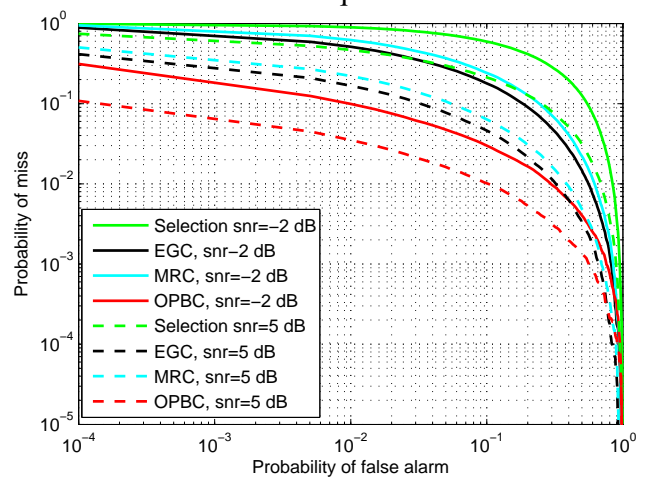

Fig. 8: Comparison of CROC curves for different values of SNR

primary signals with higher efficiency than other considered cases. From system complexity point of view, the OPBC technique appears to be more complex than other combining techniques since polarization operator is applied on per sample basis. The cost of complexity increases linearly with the number of samples $N$ as compared to SC, EGC and MRC combining techniques. In practical scenarios, cross-polarization discrimination, especially of the PUs terminals may affect the performance of this technique. Analysis of cross-polarization discrimination in the considered scenarios and investigation of suitable techniques to reduce cross-polarization discrimination need to be carried out to implement this technique practically.

\section{CONCLUSION}

In this work, the polarization domain has been exploited for SS using dual polarized antenna. The following conclusions

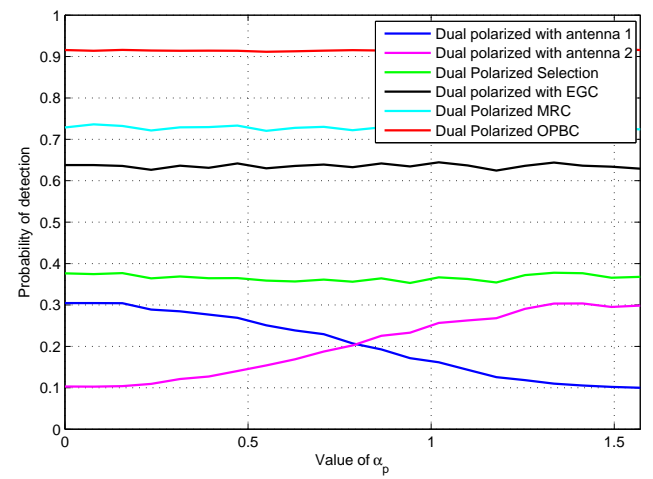

Fig. 9: Probability of detection versus value of $\alpha_{p}$ can be drawn by analyzing the simulation results. Picking any one of radiators of the dual polarized antenna provides better sensing performance than single omnidirectional antenna. SC technique provides better performance than individual cases of dual polarized antenna due to advantage in selecting the decision metric. MRC and EGC techniques provide more efficient SS due to power gain obtained by combining two signals in different ways. The OPBC technique increases SS efficiency significantly by optimally combining two signals received from branches of dual polarized antenna at the cost of system complexity. Analyzing the performance of polarization based detection in fading channels, cross-polarization discrimination of PUs terminals and the correlation of received signals in the context of dual polarized antenna can be future work in this area.

\section{REFERENCES}

[1] A. J. Poelman, "Virtual polarisation adaptation a method of increasing the detection capability of a radar system through polarisation-vector processing," in Proc. IEE Communications, Radar and Signal Processing, vol.128, pp. 261-270, 1981.

[2] F. F. Digham, M.-S. Alouini, and M. K. Simon, "On the energy detection of unknown signals over fading channels," in Proc. IEEE Int. Conf. Commun., vol. 5, pp. 3575-3579, May 2003.

[3] D. Cabric, A. Tkachenko, and R. Brodersen, "Spectrum sensing measurements of pilot, energy, and collaborative detection," in Proc. IEEE Military Commun. Conf. (MILCOM'06), pp. 1-7, Oct. 2006.

[4] B. S. Collins, "Polarization-diversity antennas for compact base stations," Microwave Journal, Vol. 43, No 1, pp. 76-88, Jan. 2000.

[5] C. B. Dietrich, K. Dietze, J. R. Nealy, and W. L. Stutzman, "Spatial, polarization, and pattern diversity for wireless handheld terminals," IEEE Trans. Antennas Propagat., vol. 49, pp. 1271-1281, 2001.

[6] S. Chatzinotas, D. Christopoulos, B. Ottersten, "Coordinated multi-point decoding with dual-polarized antennas", in proc. 7th Int. Wireless Comm. and Mobile Computing Conf. (IWCMC), pp. 157-161, July 2011.

[7] Fangfang Liu, Chunyan Feng, Caili Guo, Yue Wang and Dong Wei, "Virtual Polarization Detection: A Vector Signal Sensing Method for Cognitive Radios", in proc. IEEE 71st Vehicular Tech. Conf. (VTC 2010Spring), pp.1-5, May 2010.

[8] Fangfang Liu, Chunyan Feng, Caili Guo, Yue Wang and Dong Wei, "Polarization Spectrum Sensing Scheme for Cognitive Radios," in proc. 5th Int. Conf. on Wireless Comm., Networking and Mobile Computing (WiCom '09), pp.1-4, Sept. 2009.

[9] Dong Wei, Caili Guo, Fangfang Liu, and Zhimin Zeng, "A SINR improving scheme based on optimal polarization receiving for the cognitive radios," in proc. IEEE Int. Conf. on Network Infrastructure and Digital Content, pp. 100-104, Nov. 2009

[10] A. Goldsmith, S. Jafar, I. Maric, and S. Srinivasa, "Breaking spectrum gridlock with cognitive radios: An information theoretic perspective," in Proc. of the IEEE, vol. 97, no. 5, pp. 894-914, 2009.

[11] D. P. Stapor, "Optimal receive antenna polarization in the presence of interference and noise," IEEE Trans. on Antennas and Propagat., vol.43, no.5, pp. 473-477, May 1995.

[12] H. Urkowitz, "Energy Detection of Unknown Deterministic Signals," in Proc. IEEE, vol. 55, no. 4, pp. 523-531, April 1967.

[13] V. I. Kostylev, "Energy detection of a signal with random amplitude," in Proc. IEEE ICC, pp. 1606 - 1610, Jun. 2002.

[14] F. F. Digham, M.-S. Alouini, and M. K. Simon, "On the energy detection of unknown signals over fading channels," IEEE Trans. Commun., vol. 55, no. 1, pp. 21-24, Jan. 2007.

[15] S.P. Herath, N. Rajatheva, and C. Tellambura, "Energy Detection of Unknown Signals in Fading and Diversity Reception," IEEE Trans. Commun. vol. 59, no. 9, pp. 2443-2453, July 2011.

[16] Steven M. Kay, "Fundamentals of Statistical Signal Processing," Volume 2, Detection Theory, First Edition, 1998.

[17] J. G.Prokais, "Digital Communication," Fourth Edition, McGraw-Hill, 2001. 